\title{
Treatment of renal congestion by tolvaptan
}

\author{
Takefumi Mori $^{1} \cdot$ Takuo Hirose $^{1} \cdot$ Satoshi Kinugasa ${ }^{1}$
}

Received: 11 December 2018 / Revised: 20 December 2018 / Accepted: 21 December 2018 / Published online: 4 February 2019

(C) The Japanese Society of Hypertension 2019

Reduced renal function is associated with increased cardiovascular events and mortality, and chronic kidney disease is a risk factor for heart failure. Conversely, heart failure is a known risk factor for renal dysfunction. Thus, a vicious cycle is formed, mediated by cardio-renal functional correlations, and this cycle is commonly observed in patients with heart failure and exacerbates the prognosis of the disease [1].

Several mechanisms have been reported to be involved in the cardio-renal connection. Notably, vasoconstrictive neurohormones including the renin-angiotensin system, vasopressin and catecholamines are known to play a major role. In heart failure, arterial underfilling stimulates these neurohormones, which induce renal ischemia, hypoxia, and $\mathrm{Na}$ retention and enhance volume overload.

In this regard, a recent report by Chiba et al. [2] examined the mechanism of the cardio-renal connection using Dahl salt-sensitive rats and a model of hypertensive cardiorenal failure. They conducted a study in which renal hemodynamic changes were measured during the induction of heart failure. Local renal blood flow (RBF) was measured by renal ultrasound, and renal medullary pressure was measured using a fiber-optic pressure sensor. The resistance index and venous impedance index were significantly increased in response to hypertensive cardio-renal failure by a high salt diet, suggesting that renal medullary blood flow was reduced.

Due to its vascular and tubular anatomy, the kidney is hypoxic, especially in the renal medulla. The medulla is actively rendered hypoxic by a countercurrent mechanism, in which oxygen diffuses from arteries to veins that run

Takefumi Mori

tmori@tohoku-mpu.ac.jp

1 Division of Nephrology and Endocrinology, Tohoku Medical and Pharmaceutical University, 1-15-1, Fukumuro, Miyagino, Sendai 983-8536, Japan parallel to each other. The oxygen pressure at the deep renal medulla can be as low as $10-20 \mathrm{mmHg}$ [3].

The collecting duct and the thin limb of Henle, which are major tubules in the inner medulla, do not actively transport sodium and thus do not require much oxygen. In contrast, the proximal tubules and the thick ascending limb in the outer medulla actively transport sodium and require large amounts of oxygen, causing the outer medulla to become the most vulnerable region to hypoxia. $\mathrm{Na}-\mathrm{K}-2 \mathrm{Cl}$ cotransporters exist in the luminal side of the thick ascending limb, whose activity is dependent on the concentration of sodium, which is pumped out of the tubules by basolateral Na-K ATPases. The Na-K ATPase activity is determined by oxygen-consuming mitochondrial ATP production. In addition, medullary blood flow is only $10 \%$ or less of the total RBF, and even small changes in renal medullary blood flow can induce hypoxia in the medulla [4]. Therefore, an imbalance between renal medullary blood flow and oxygen consumption could cause severe hypoxia in the outer medulla. It has been reported that the outer medulla is where a tubulointerstitial injury is initially observed in animal models of ischemic renal diseases, such as hypertension, diabetes and ischemic acute kidney injury [5].

Renal medullary circulation is important in the pathogenesis of cardio-renal failure. Using laser Doppler flowmetry in rats, it has been shown that medullary blood flow, as well as total or cortical blood flow, is not autoregulated during changes in renal arterial perfusion pressure [4]. Thus, reduction of the renal perfusion pressure due to arterial underfilling during heart failure results in the reduction of medullary blood flow and enhances outer medullary ischemia and hypoxia.

Renal medullary circulation is also involved in the regulation of $\mathrm{Na}$ and fluid volume [4, 5]. A mere 10-30\% reduction in medullary blood flow results in increased $\mathrm{Na}$ retention in the renal tubules. A reduction of medullary blood flow in heart failure induces further increases in $\mathrm{Na}$ retention and volume overload [5].

Moreover, renal venous congestion could also contribute to the pathogenesis of cardio-renal failure [5]. It has been 


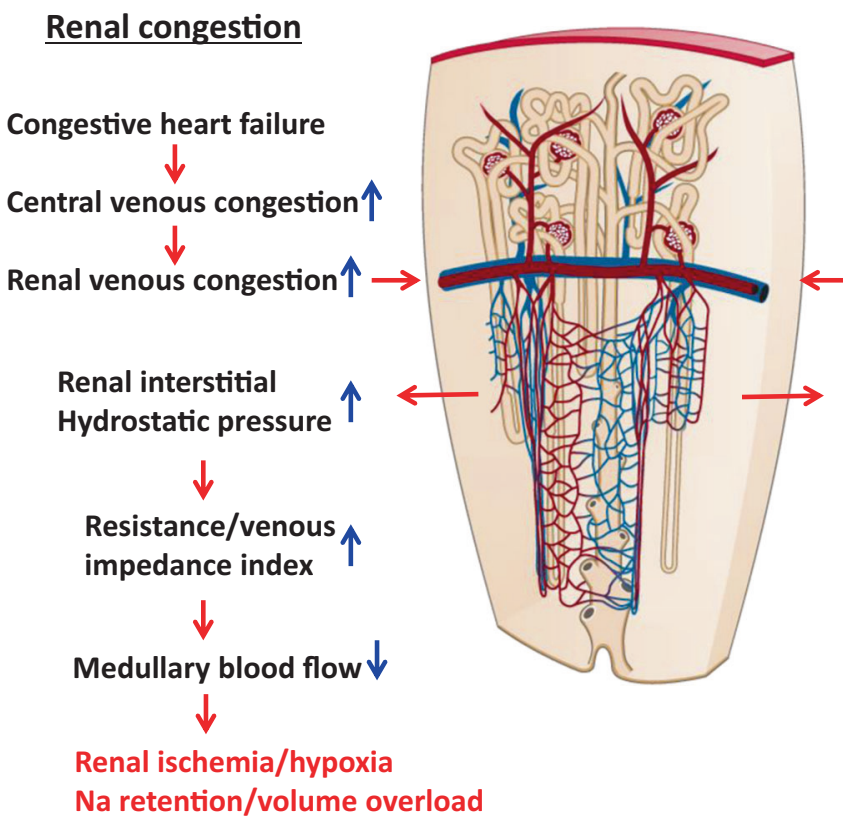

Tolvaptan treatment

Improve heart failure

Central venous congestion $\downarrow$

$\downarrow$

Renal venous congestion $\downarrow$

Renal interstitial

Hydrostatic pressure $\downarrow$

$\downarrow$

Resistance/venous

impedance index

$\downarrow$

Medullary blood flow $\uparrow$

$\downarrow$

Renal protection

$\mathrm{Na}$ excretion/volume control

Fig. 1 Hypothesis for renal congestion and tolvaptan treatment. Congestive heart failure induces central/renal venous congestion and increases renal interstitial hydrostatic pressure (RIHP). The increase in RIHP reduces medullary blood flow (MBF) by increasing the resistance and venous impedance indices. The reduction of MBF results in renal ischemia and Na retention. In contrast, treatment with tolvaptan improves heart failure and reduces central/renal venous congestion and RIHP. The reduction of RIHP improves the resistance and venous impedance indices and MBF, which could improve renal protection and $\mathrm{Na}$ excretion

demonstrated that renal venous congestion may regulate $\mathrm{RBF}$ and $\mathrm{Na}$ retention. The key factors that explain these mechanisms are medullary blood flow and interstitial pressure. Renal interstitial fluid is produced by the medullary tubules, and one of the upstream origins is reabsorption from the collecting ducts to the venous capillaries in the cortex. Since the kidney is surrounded by a tight capsule, congestion of the renal interstitial flow and interstitial pressure can be increased by central or renal venous congestion. An increase in interstitial pressure could lead to the reduction of medullary blood flow and glomerular filtration rate (GFR) due to the compression of venous capillaries, such as the vasa recta, and tubules, respectively [6].

Medullary blood flow and GFR affect $\mathrm{Na}$ and water excretion during volume overload, and renal venous congestion reduces $\mathrm{Na}$ and water excretion through the inappropriate reduction of renal medullary blood flow and GFR.

Increased central venous pressure (CVP) and renal venous pressure (RVP) are observed in congestive heart failure patients compared to healthy subjects. Increased CVP is associated with GFR and mortality. During advanced decompensated chronic heart failure (CHF), increased CVP, rather than the cardiac index, correlates with the future reduction of GFR after discharge from the hospital. Therefore, renal venous hypertension could play a role in the pathogenesis of renal failure during heart failure. It has been shown that high RVP induces increased renal interstitial hydrostatic pressure (RIHP) and the reduction of GFR.
Although furosemide has been widely used for controlling fluid volume in heart failure, there are several concerns over the use of this drug. First, vasoconstrictive mediators, such as the renin-angiotensin system, vasopressin and catecholamine, can be enhanced. Second, metabolic disturbances, such as hyperammonemia, and electrolyte disorders, such as hyponatremia, hypokalemia, hypocalcemia and hypomagnesemia, can be seen. Third, a rebound of $\mathrm{Na}$ reabsorption can be observed, since furosemide is a shortacting drug and does not last $24 \mathrm{~h}$. Finally, venous capillary collapse can be observed despite remaining renal congestion.

Tolvaptan has been used for volume control in heart and liver failure in Japan. RBF is reduced when heart failure patients are treated with furosemide, but the decrease is improved by switching to tolvaptan [7]. There are several mechanisms that could explain the differences between the two diuretics. First, neuro-hormonal activations are different between the two drugs. While furosemide strongly stimulates plasma renin activity, catecholamine and vasopressin, a Japanese phase 3 study has shown that plasma renin activity and vasopressin are not significantly increased after treatment with tolvaptan in heart failure patients [8]. Second, tolvaptan can maintain $\mathrm{RBF}$ by retaining intracapillary volume. As shown in Fig. 1, tolvaptan increases free water excretion and crystal osmolality, which could induce volume intake into the capillaries from the third space. However, crystal osmolytes, such as sodium, do not remain 
inside the capillaries and eventually diffuse into the interstitium. Additionally, colloid osmolytes, such as albumin, can pull water into the capillary. These two forces enable the maintenance of RBF, despite the reduction of total body water. We have previously reported that intracellular to extracellular movement of water after tolvaptan treatment in patients with cardio-renal failure was observed using bioimpedance analysis [9].

Despite these clinical observations, the precise mechanism of renal hemodynamic changes by tolvaptan remains to be elucidated. The report by Chiba et al. demonstrated the effects of tolvaptan on renal hemodynamics in Dahl saltsensitive rats. Rats treated with an $8 \%$ salt diet developed hypertensive heart failure and renal dysfunction. Renal medullary pressure and CVP were significantly reduced by treatment with tolvaptan. The increased local renal resistance index after salt loading was improved by tolvaptan treatment. Interestingly, renal dysfunction and renal fibrosis were also improved. Although plasma renin activity was significantly increased, the plasma aldosterone concentration and renal tissue noradrenaline level were not increased. This was consistent with the report by Morooka et al., which showed that tolvaptan improved glomerular and tubulo-interstitial injury in high-salt diet Dahl salt-sensitive rats [10]. In this study, the mRNA expressions of renin and vasopressin type 1a (V1) and type 2 (V2) receptors were reduced.

Vasopressin binds to both V1a and V2 receptors in the kidney. V2 receptors induce water diuresis, while V1a receptors reduce medullary blood flow. Thus, the blockade of V2 receptors by tolvaptan may increase vasopressin binding to V1a receptors and reduce medullary blood flow. However, this was not observed in the present study, possibly due to the reduction of V1a expression.

In contrast to the study by Morooka et al., Chiba et al. demonstrated a reduction in systolic blood pressure, which does not often occur in human studies. This could lead to the reduction of renal fibrosis, since blood pressure is a major factor for renal injury in Dahl salt-sensitive rats. However, systolic blood pressure was not correlated with renal fibrosis in the current study, so it is unlikely that this is the mechanism underlying the renoprotection by tolvaptan. Rather, plasma renin activity was negatively correlated with fibrosis. Plasma renin activity was suppressed after salt loading in Dahl salt-sensitive rats, in response to the volume expansion. Therefore, the increase in plasma renin activity may be due to the volume reduction induced by tolvaptan. Since it has been shown that the local renal renin angiotensin system is responsible for renal injury in Dahl saltsensitive rats, further investigation is required.

Dahl salt-sensitive rats develop hypertension with high salt loading, resulting in heart and renal failure [11]. High salt loading in Dahl salt-sensitive rats reduces renal medullary blood flow and increases $\mathrm{Na}$ reabsorption. Enhanced NADPH oxidase and reduced NOS activity in the medulla are involved in the reduction of renal medullary blood flow. Increasing nitric oxide (NO) by the intramedullary infusion of L-arginine, or infusion of the superoxide dismutase mimetic TEMPOL, reduces superoxide ameliorated salt-sensitive hypertension in these rats [11].

It has been shown that reactive oxygen species (ROS) and NO are produced in the medullary thick ascending limb of Henle, and diffuse into pericytes surrounding the vasa recta, which is the main vessel that regulates medullary blood flow. Pericytes are smooth, muscle-like cells that can constrict the vasa recta and regulate medullary blood flow independent of total renal flow. Medullary blood flow is regulated by the balance between superoxide and NO diffusion from the thick ascending limb of Henle into the pericytes of the vasa recta [11].

The enhanced local renin-angiotensin system in Dahl salt-sensitive rats could produce ROS in the renal medulla, reduce renal medullary blood flow and increase $\mathrm{Na}$ retention. Thus, the increase in the resistance index observed by Chiba et al. could be due to increased ROS in the renal medulla.

We recently reported that an increase in RIHP and congestion of the intrarenal capillaries were involved in the pathogenesis of renal injury in a novel rat model of renal venous congestion by ligating the inferior vena cava [6] Renal congestion-induced renal injury was improved when RIHP was reduced by renal decapsulation. In renal congestion, pericytes of the vasa recta were detached, and then a transition into myofibroblasts was observed. This was also partially reduced by renal decapsulation.

How does tolvaptan protect the kidneys from injury in Dahl salt-sensitive rats? First, although renin activity was increased, the plasma aldosterone concentration was not increased. This could be an initial mechanism to explain the effect of tolvaptan. Second, volume loss by tolvaptan could improve renal congestion. Third, tolvaptan blocks V2 receptors and inhibits water reabsorption by aquaporin. Since water reabsorption by aquaporin occurs upstream of the interstitial fluid, it increases the RIHP. Thus, treatment by tolvaptan could reduce RIHP by reducing interstitial fluid both upstream and downstream. This is consistent with the results by Chiba et al. that renal medullary pressure was reduced by treatment with tolvaptan in Dahl salt-sensitive rats.

In summary, the manuscript by Chiba et al. presented a novel possibility that tolvaptan improves renal medullary circulation in Dahl salt-sensitive rats with a model of renal congestion. The overall mechanism involved in the present study is summarized in Fig. 1. Although the precise mechanism is unknown, it is possible that the improvement of renal hypoxia by the removal of renal congestion by 
tolvaptan plays an important role in the reduction of renal injury. Further investigation is required regarding whether RIHP and the pericyte-myofibroblast transformation are involved in the renal protection by tolvaptan.

\section{Compliance with ethical standards}

Conflict of interest TM is a consultant for a clinical trial being conducted by Otsuka Pharmaceutical. TM has also received honoraria for lectures and research funding from Otsuka Pharmaceutical. The remaining authors declare that they have no conflict of interest.

Publisher's note: Springer Nature remains neutral with regard to jurisdictional claims in published maps and institutional affiliations.

\section{References}

1. Damman K, Testani JM. The kidney in heart failure: an update. Eur Heart J. 2015;36:1437-44.

2. Chiba H, Seo Y, Sai S, Namekawa M, Ishizu T, Aonuma K. Renoprotective effects of tolvaptan in hypertensive heart failure rats depend on renal decongestion. Hypertens Res. 2018. https:// doi.org/10.1038/s41440-018-0169-3 [Epub ahead of print].

3. Zou AP, Cowley AW Jr. Reactive oxygen species and molecular regulation of renal oxygenation. Acta Physiol Scand. 2003;179:233-41.

4. Cowley AW Jr. Role of the renal medulla in volume and arterial pressure regulation. Am J Physiol. 1997;273:R1-15.
5. Mori T, Ohsaki Y, Oba-Yabana I, Ito S. Diuretic usage for protection against end-organ damage in liver cirrhosis and heart failure. Hepatol Res. 2017;47:11-22.

6. Shimada S, Hirose T, Takahashi C, Sato E, Kinugasa S, Ohsaki Y et al. Pathophysiological and molecular mechanisms involved in renal congestion in a novel rat model. Sci Rep. 2018;8: 16808.

7. Costello-Boerrigter LC, Smith WB, Boerrigter G, Ouyang J, Zimmer CA, Orlandi C et al. Vasopressin-2-receptor antagonism augments water excretion without changes in renal hemodynamics or sodium and potassium excretion in human heart failure. Am $\mathbf{J}$ Physiol Renal Physiol. 2006;290:F273-8.

8. Matsuzaki M, Hori M, Izumi T, Fukunami M, Tolvaptan I. Efficacy and safety of tolvaptan in heart failure patients with volume overload despite the standard treatment with conventional diuretics: a phase III, randomized, double-blind, placebo-controlled study (QUEST study). Cardiovasc Drugs Ther. 2011;25(Suppl 1): S33-45.

9. Mori T, Oba I, Koizumi K, Kodama M, Shimanuki M, Tanno M et al. Beneficial role of tolvaptan in the control of body fluids without reductions in residual renal function in patients undergoing peritoneal dialysis. Adv Perit Dial. 2013;29:33-7.

10. Morooka H, Iwanaga Y, Tamaki Y, Takase T, Akahoshi Y, Nakano $\mathrm{Y}$ et al. Chronic administration of oral vasopressin type 2 receptor antagonist tolvaptan exerts both myocardial and renal protective effects in rats with hypertensive heart failure. Circ Heart Fail. 2012;5:484-92.

11. Mori T, Ogawa S, Cowely AW Jr., Ito S. Role of renal medullary oxidative and/or carbonyl stress in salt-sensitive hypertension and diabetes. Clin Exp Pharmacol Physiol. 2012;39:125-31. 